\title{
Exploring Bottom-Up Visual Processing and Visual Hallucinations in Parkinson's Disease With Dementia
}

\begin{abstract}
Nicholas Murphy 1,2, Alison Killen ${ }^{1}$, Rajnish Kumar Gupta ${ }^{3}$, Sara Graziadio ${ }^{4}$, Lynn Rochester ${ }^{1}$, Michael Firbank ${ }^{1}$, Mark R. Baker ${ }^{1}$, Charlotte Allan ${ }^{1}$, Daniel Collerton ${ }^{1}$, John-Paul Taylor ${ }^{1}$ and Prabitha Urwyler ${ }^{1,3,5,6 *}$

${ }^{1}$ Faculty of Medical Sciences, Translational and Clinical Research Institute, Newcastle University, Newcastle upon Tyne, United Kingdom, ${ }^{2}$ Menninger Department of Psychiatry and Behavioral Sciences, Baylor College of Medicine, Houston, TX, United States, ${ }^{3}$ ARTORG Center for Biomedical Engineering Research, University of Bern, Bern, Switzerland, ${ }^{4}$ National Institute for Health Research Newcastle In Vitro Diagnostics Co-operative, Newcastle Upon Tyne Hospitals Foundation Trust, Newcastle upon Tyne, United Kingdom, ${ }^{5}$ Gerontechnology and Rehabilitation Group, University of Bern, Bern, Switzerland,

${ }^{6}$ University Neurorehabilitation Unit, Department of Neurology, Inselspital, Bern University Hospital, Bern, Switzerland
\end{abstract}

Visual hallucinations $(\mathrm{VH})$ are a common symptom of Parkinson's disease with dementia (PDD), affecting up to $65 \%$ of cases. Integrative models of their etiology posit that a decline in executive control of the visuo-perceptual system is a primary mechanism of $\mathrm{VH}$ generation. The role of bottom-up processing in the manifestation of $\mathrm{VH}$ in this condition is still not clear although visual evoked potential (VEP) differences have been associated with $\mathrm{VH}$ at an earlier stage of PD. Here we compared the amplitude and latency pattern reversal VEPs in healthy controls $(n=21)$ and PDD patients $(n=34)$ with a range of $\mathrm{VH}$ severities. PDD patients showed increased $\mathrm{N} 2$ latency relative to controls, but no significant differences in VEP measures were found for patients reporting complex $\mathrm{VH}(\mathrm{CVH})(n=17)$ compared to those without $\mathrm{VH}$. Our VEP findings support previous reports of declining visual system physiology in PDD and some evidence of visual system differences between patients with and without $\mathrm{VH}$. However, we did not replicate previous findings of a major relationships between the integrity of the visual pathway and $\mathrm{VH}$.

Keywords: visual processing, Parkinson's disease dementia, visual hallucination, Lewy body, visual evoked potential

\section{INTRODUCTION}

Visual symptoms are common in Parkinson's disease (PD), and include double vision, dry or painful eyes, poor contrast sensitivity, problems with color vision, and blurring of vision or lowered acuity $(1-5)$. In $45 \%$ of PD cases without dementia $(6,7)$, and up to $65 \%$ of cases with dementia (PDD) (8), patients will also experience visual hallucinations (VH). The early presence of $\mathrm{VH}$ is a strong predictor of cognitive decline (9), as well as increased mortality and overall reduced quality of life for patients and their carers $(10,11)$.

Visual dysfunction in PD has been linked to the physical decline of retinal function over the course of disease development due to depletion of retinal dopamine (12), and retinal nerve fiber layer thinning (13). Electrophysiological measures of visual health, such as the visual evoked 
potential (VEP), and the electroretinogram (ERG), have been widely used to support the diagnosis of PD as indirect measures of health and integrity of early bottom-up visual processing pathways. Measurements of scalp potentials, as well as scotopic alpha and beta waves generated on the retina during foveal stimulation typically demonstrate a slowing of peak activity in PDD patients relative to controls (14-16), acting as indirect support for pathological evidence of a decline in pre-geniculate visual function $(12,13)$.

Models of VH in Lewy body dementias [including dementia with Lewy bodies (DLB), and PDD] have posited that VH are a product of the inefficient integration of multiple perceptual subdivisions of the visual system including retinal input $(17,18)$. Healthy visual perception involves the prediction of sensory inputs expected from the salient features of images (based upon long-term memory of similar images and current context) which are then matched to the actual sensory inputs to minimize any discrepancy between the two. Thus, perception needs to balance predictions and sensory information. Impairments in cognitive control across executive networks in PDD lead to difficulties balancing these processes, thus impairing the accuracy of matching the visual input to expectations. Despite the precise etiology of $\mathrm{VH}$ being unclear, variations in the frequency of $\mathrm{VH}$ over the course of disease progression suggests that these hallucinations reflect a complex relationship between declining sensory function and dysfunctional predictions (17-22).

In this investigation we sought to characterize the components of early bottom-up processing in PDD patients, using the pattern reversal visual evoked potential, and to relate the response features to the complexity of the VHs experienced. Based on available evidence of physiological decline in PDD we predicted that we would observe a general reduction in the amplitude of the VEP components, as well as an increase in the P1 latency (23). In addition, we expected baseline visual acuity and visual perception, to demonstrate a decline in those with a more severe and frequent complex VH (CVH). This should also extend to an association between VEP P1 and N2 measurements with $\mathrm{VH}$ experience, as both of these are thought to be contingent upon attentional and perceptual processes $(24,25)$, which are, in particular, disrupted by Lewy body pathology $(18,26)$.

\section{METHODS}

\section{Participants}

A total of 21 healthy controls, and 38 Parkinson's disease with dementia (PDD) patients were recruited from the North East of England. Ethical approval was granted by the Newcastle National Health Service (NHS) Health Research Authority (HRA) (REC reference: 13/NE/0252; R\&D reference: 6691). The diagnosis of PDD was confirmed by two independent and experienced clinicians (Charlotte Allan, John-Paul Taylor) and met with the standards described in the international PD diagnostic criteria (27). Participants were excluded from the study if baseline assessment revealed the presence of comorbid factors including stroke, non-PD related dementia, and/or visual dysfunction secondary to glaucoma. All procedures related to the study were explained to the participants and written informed consent was obtained prior to participation.

\section{Clinical Assessments}

All participants were assessed on their level of global cognitive function using the Mini Mental State Exam [MMSE, (28); maximum score of 30] and the Cambridge Cognitive Test Battery [CAMCOG total score $(29,30)$; maximum score of 107]. Motor function was assessed using the total (left and right) score from the Unified Parkinson's disease rating scale section three [UPDRS-III (31); maximum score of 57]. Measurements of fluency and executive functioning were derived from the category fluency test (32) and Trail Making Test A [TMT-A (33)].

The integrity of the participant's visual acuity was assessed using a detailed screening questionnaire, computerized Freiburg acuity testing (34), and the LOGMAR (Logarithm of the Minimum Angle of Resolution) scale of visual acuity. Visuoperceptual function was assessed using performance on motion sensitivity (35), angle discrimination (35), and performance on the pareidolic imagery test (36).

\section{Visual Hallucinations}

The hallucination subscale (frequency 0 to 4 , severity: 0 to $3-$ not applicable, mild, moderate, marked, and level of distress 0 to 5) of the Neuropsychiatric Inventory (NPIHal) (37) was used for assessing $\mathrm{VH}$ occurring in the previous month, with the NPIHal score (frequency $\times$ severity of hallucinations) derived as a measure. The NPI-Hall score used in this manuscript is calculated by multiplying the NPI hallucination frequency with NPI hallucination severity. The frequency of hallucination in NPI is coded as 0 -not applicable, 1 -occasionally (less than once per week), 2-often (about once per week), 3-frequently (several times per week), and 4-very frequently (once or more per day). Severity of hallucination with NPI is scored as 0 -not applicable, 1-mild, 2-moderate and 3-marked. For reliability, patients and carers were independently asked about the occurrence of $\mathrm{VH}$ in the month before using the North-East Visual Hallucinations Interview (NEVHI) (38). Any discrepancies in the reporting of VH (39) were discussed with both parties and the assessor, with reformulation of NPIHal scores (wherever the patient seemed to lack insight, primacy was given to caregiver opinion).

Participants were classed as active complex visual hallucinators (PD-CVH, $n=17$ ) if they had complex VH $(\mathrm{CVH})$ in the month preceding their interview; otherwise, they were classed as non-hallucinators [controls $(n=21)$ and PD-NCVH $(n=17)$ ]. Participants with minor VH (e.g., passage or feeling of presence) but no complex VH in the last month were included in the PD-NCVH group. This distinction was made due to the different etiologic basis to $\mathrm{CVH}$ even though minor $\mathrm{VH}$ typically precede CVH. Patients in this study map onto the same categories used in previously published research from our lab [see (40)]. Additional analysis with a more stringent grouping of PDD-VH (including complex, minor, presence, passage, simple) and PDD-NVH are provided at a later stage in this manuscript. 


\section{EEG}

\section{Visual Evoked Potential Presentation and Recording}

The VEP adhered to the specifications proposed by the International Society for Clinical Electrophysiology of Vision (41) (ISCEV). Participants viewed a black and white checkerboard pattern whilst the checks (visual angle of $0.6^{\circ}$ ) reversed phase at a rate of $1 \mathrm{~Hz}$ (switching to the opposite phase every $500 \mathrm{~ms})$, for 200 sweeps, with a brief rest period $(3,000 \mathrm{~ms})$ after 100 sweeps. During stimulus presentation a pink dot was placed in the center of the display as a focus point, which the participant was instructed to look at. This was intended to prevent wandering gaze during the check reversal and was presented on top of a gray background during the rest period. The stimulus was generated on a Dell OptiPlex 755 (Microsoft Windows XP) using Matlab v2012a (The MathWorks, 2012), and presented using a Dell U2412M 24-inch LCD monitor (resolution: $1920 \times 1,200$ pixels refresh rate: $60 \mathrm{~Hz}$ ). Pattern reversal VEPs were recorded during three separate viewing conditions (both eyes, left eye, right eye), using an ASA-LAB 136 system amplifier and the ASA-LAB recording software (version 4.9.1) in combination with a $128 \mathrm{Ag} / \mathrm{AgCl}$ channel Waveguard cap [10-5 system (42), Advanced Neuro Technologies]. The ground electrode was placed on the right clavicle, and $\mathrm{Fz}$ was used as the reference electrode. Electrode impedance was kept below $5 \mathrm{k} \Omega$, and no filters were applied during the acquisition of EEG data.

\section{Pre-processing}

Signal processing and measurement was performed using Matlab v2012a (The MathWorks, 2012), with the EEGLab (43), ERPLab (44), and current source density (45) (CSD) toolboxes. Individual sweeps were split into epochs of $400 \mathrm{~ms}$, a baseline period of $100 \mathrm{~ms}$ prior to stimulus presentation, and a post-stimulus period of $300 \mathrm{~ms}$. Epochs were baseline corrected using the mean of the data in the pre-stimulus period and filtered using a 0.1 to $45 \mathrm{~Hz}$ bandpass filter. Individual channels with a kurtosis value greater than three standard deviations from the capwide mean were removed and recreated after pre-processing using spherical interpolation $(43,46-48)$. After removing trials containing blinks, muscular activity, and drifting potentials (impedance related artefacts), broad spatial effects of the electric field were attenuated by applying a Laplacian transform (45). This approach was applied to reduce the likelihood of false positives in spatially distant locations when defining the occipital region of interest (ROI).

\section{Measurement}

To account for individual variance in the timing of synaptic communication the VEP components were measured within windows defined by the global field power (GFP) for each individual. The VEP components elicited three GFP maxima following stimulus presentation, each of which was used as the center point for the corresponding component window (GFP maxima $\pm 10 \mathrm{~ms}$ ). The occipital ROI was defined by measuring the amplitudes of the P1 component for the grand average of the control data set and using the 20 electrodes with the greatest amplitude as the limit for the ROI. Individual subject measurements of peak latency and mean amplitude were taken from the average VEP waveform within the occipital ROI. To account for potential inter-ocular latency differences, we estimated the difference between $\mathrm{P} 1$ peak latency measurements for the left and right eyes. Three separate recordings were taken from each participant: viewing with both eyes; viewing with left eye; viewing with right eye. The three recordings were used to help screen for inter-ocular variations that could have indicated undocumented eye disease. The analyses were performed using the both eyes recording.

\section{Statistical Analysis}

All statistical tests were performed using the Statistical Package for the Social Sciences (SPSS, version 22). For all comparisons the data were inspected for violations of normality using the Shapiro-Wilk test due to it being more sensitive to small sample sizes. Demographic and baseline factors were compared using independent samples $t$-tests. We compared the measurements of amplitude and latency separately for each component using univariate analysis of variance controlling for age and interocular latency difference between the left and right eyes. As further validation of the findings, we expanded our model to include UPDRS, CAMCOG, and L-Dopa dose as covariates. Effect sizes were estimated using the partial eta squared measure $\left(\eta^{2}\right)$. To explore the relationship between the variance within our physiological measurements and $\mathrm{VH}$ experience in the hallucinating PDD group, we performed parametric correlations between each VEP measurement and NPI hallucination score (NPIHal) and NPI hallucination severity. To help identify any variance in our measurements accounted for by clinical and/or demographic factors we performed additional parametric correlations between the VEP measurements and each value. To investigate the contribution of the minor $\mathrm{VH}$, all the abovementioned tests were explored with regrouping PDD-NVH vs. PDD-VH. Significance for all tests was determined using an alpha criterion of $p<0.05$, and Bonferroni corrected for multiple comparisons (corrected alpha criterion of $p<0.016$ ). Where appropriate un-corrected correlations are reported to highlight trends within individual results.

\section{RESULTS}

\section{Demographics and Clinical Scores}

Demographic results are summarized in Table 1. All groups were matched for age and there were no significant differences in duration of $\mathrm{PD}(p=0.09)$ or levodopa dose $(p=0.27)$ between the PDD-CVH and PDD-NCVH groups. PDD patients displayed a significant reduction in global cognitive function, UPDRS motor score relative to controls, with the PDD-CVH group global cognitive function and motor function were significantly worse when compared to the PDD-NCVH group. The NPI recorded the severity of hallucinations as mild in $29.4 \%$ (5 of 17) PDD$\mathrm{NCVH}$ which were phenomenologically classified as Illusions (3 of $5,75 \%$ ), presence ( 4 of $5,66.7 \%$ ), shadow ( 3 of $5,75 \%$ ), and simple $(3$ of $5,75 \%)$ by the NEVHI. Further stringent grouping into PDD_NVH dropped the reporting of mild hallucinations to $10 \%$. 
TABLE 1 | Participant demographics and clinical scores.

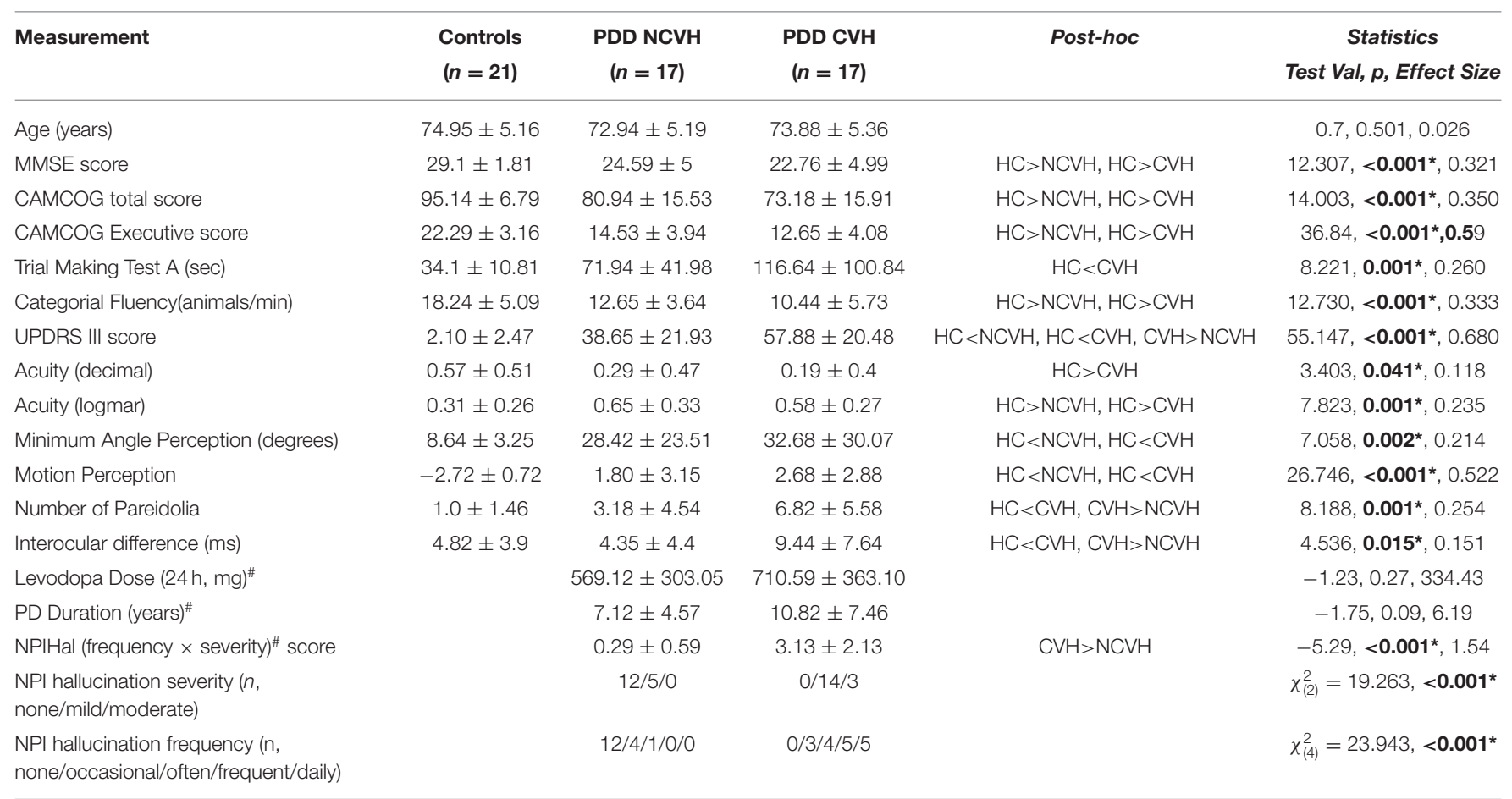

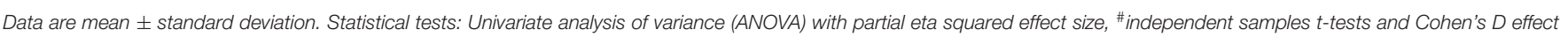

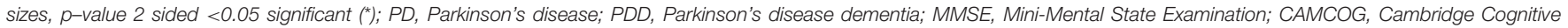

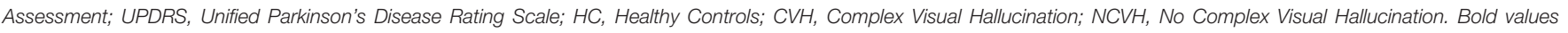
indicate statistically significant values.

\section{Visual Integrity and Visual Perceptual Scores}

Cataracts were reported by $20 \%$ (Controls $6.9 \%$, PDD-NCVH $3.4 \%$, PDD-CVH $10.3 \%$ ) of the recruited participants while $19 \%$ of the participants had cataracts removed (Controls 5.2\%, PDD$\mathrm{NCVH}$ 6.9\%, PDD-CVH 6.9\%). Other ophthalmological history reported include glaucoma by two participants (PDD-VH 1.7\%, HC $1.7 \%$ ), macular degeneration by one participant (HC 1.7\%), and laser treatment (HC 1.7\%).

Visual acuity and perceptual scores are summarized in Table 1. There was a pattern of overall decline in visual integrity within the PDD patients relative to the control group, characterized by a significant reduction in LOGMAR $(p=0.001)$ measurements of visual acuity. As expected, PDD-CVH patients showed a characteristic significant increase in the number of false perceptions $(p=0.047)$ reported during the pareidolia task compared to $\mathrm{PDD}-\mathrm{NCVH}$ patients. Interocular latency differences were significantly higher in PDD-CVH compared to the PDD-NCVH $(p=0.028)$ group.

\section{Visual Evoked Potential}

Amplitude tended to be smaller, and latency later in PDD vs controls (Amplitude N1: $-0.844 \pm 0.67$ vs. $-1.27 \pm 0.93$, P1: $2.34 \pm 1.57$ vs. $3.61 \pm 2.55, \mathrm{~N} 2:-1.12 \pm 1.02$ vs. $-1.64 \pm$ 1.44; Latency N1: $91.79 \pm 9.60$ vs. $88.28 \pm 8.62$; P1: 127.21 \pm 6.96 vs. $124.50 \pm 7.31, \mathrm{~N} 2: 175.54 \pm 14.84$ vs. 162.26 \pm 8.98 ), although this was only significant for P1 and $\mathrm{N} 2$ components. A visual representation of the VEP is presented in
Figure 1. There were no significant differences between PDD$\mathrm{CVH}$ vs. PDD-NCVH. (see Figure 2) for the VEP latencies and amplitudes (N1: $p=0.210 ; \mathrm{P} 1: p=0.120$ ), except N2 latency (Controls: $162.27 \pm 8.98$, PDD-NCVH: $176.93 \pm 14.69$, PDDCVH:174.15 $\left.\pm 15.30, F=7.95, p=0.001, \eta^{2}=0.241\right)$. A post hoc Bonferroni comparison indicated that $\mathrm{N} 2$ latency in controls was significantly less than PDD-NCVH group $(p=0.02)$ and PDD-CVH $(p=0.018)$, but the N2 latency did not differ between the CVH and NCVH group while controlling for age and interocular latency differences. Further validation result indicates no significant effect between PDD_NCVH and PDD_CVH on latency and amplitude of N1, P1, and N2 components even after controlling for UPDRS, CAMCOG, and L-Dopa dose along with age and interocular latency.

Additional analysis with a stringent regrouping of PDD-NVH and PDD-VH show similar results (Table 2) as in Figures 1, 2. Post hoc Bonferroni comparison indicated that N2 latency in controls was significantly less than PDD-NVH group $(p=0.029)$ and PDD-VH $(p=0.001)$, but the N2 latency did not differ between the $\mathrm{VH}$ and $\mathrm{NVH}$ group. The regrouping of minor $\mathrm{VH}$ into the PDD-VH group shows a trend toward significant difference for the N1 $(p=0.072)$ and $\mathrm{P} 1(p=0.099)$ amplitude.

\section{Clinical Correlations}

Clinical correlation of VEP components for latency and amplitude are shown in Tables 3, 4, respectively. Hallucination experience as quantified using the NPIHal subscale score (frequency $x$ severity) was not significantly related to the 


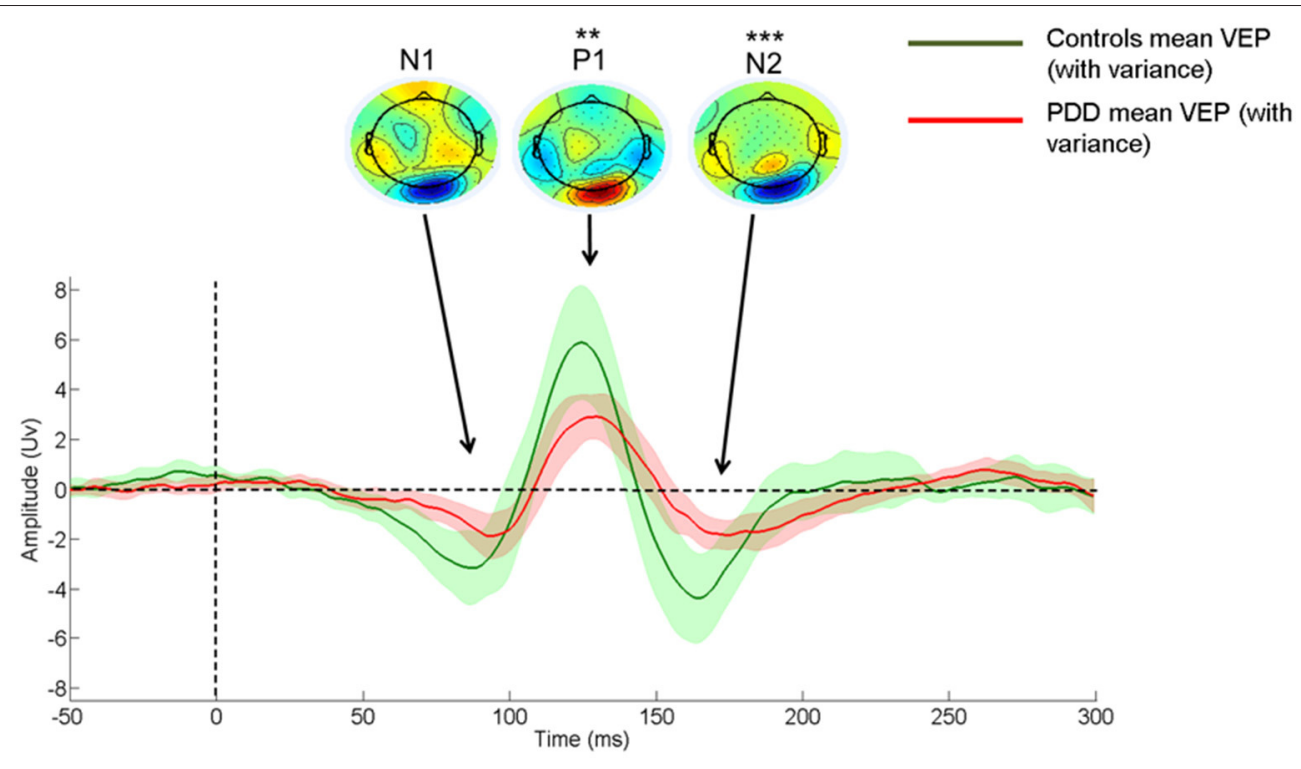

${ }^{* *}$ Amplitude significantly different at $p<0.05$ )

*** Latency significantly different at $p<0.05$ )

FIGURE 1 | Comparison of Control and Patient VEP waveform. PDD, Parkinson's disease dementia; VEP, Visual evoked potential; VEP components - N1, P1, and N2.
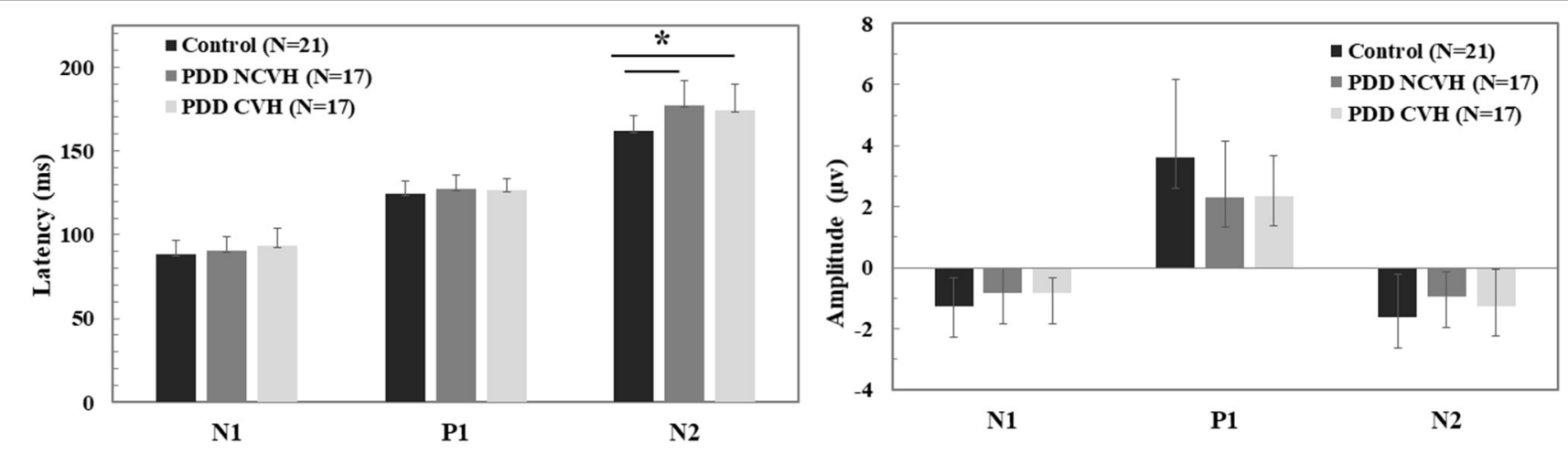

FIGURE 2 | Comparison of the visual evoked potential component (N1, P1, and N2) amplitude and latency. Statistical tests: Univariate analysis of variance (ANOVA), $\mathrm{df}=52$, $p$-value 2 sided $<0.05$ significant; PDD, Parkinson's disease dementia; HC, Healthy Controls; CVH, Complex Visual Hallucination; NCVH, No Complex Visual Hallucination; ${ }^{*}$ Post-hoc $=\mathrm{CVH}>\mathrm{HC}, \mathrm{NCVH}>\mathrm{HC}$.

measurements of any of the VEP components. However, $\mathrm{VH}$ severity assessed using the NPI hallucination severity showed significant negative correlation with P1 latency in PDD-NCVH patients $(r=-0.492, p=0.045)$ and $\mathrm{P} 1$ amplitude in PDD-CVH patients $(r=-0.555, p$ $=0.021$ ).

Minimum angle of perception demonstrated significant negative correlation with $\mathrm{N} 1(r=-0.407, p=0.028)$ and N2 $(r=-0.487, p=0.007)$ latency in PDD patients and was also significant for N1 latency in PDD-CVH patients $(r$ $=-0.648, p=0.012$ ). False perception in Pareidolia tasks demonstrated significant positive correlation with $\mathrm{N} 1$ amplitude in PDD-CVH $(r=0.504, p=0.039)$ patients. Interocular latency showed no significant correlations with NPI hallucination severity $(r=-0.280, p=0.220)$, and NPI hallucination frequency $(r=-0.119, p=0.606)$.

Executive scores such as CAMCOG Executive demonstrated significant correlations with $\mathrm{N} 1$ amplitude in PDD patients $(r=$ $-0.370, p=0.031)$ and PDD-NCVH patients $(r=-0.564, p=$ $0.018)$, TMT-A with P1 latency in PDD-NCVH $(r=0.555, p=$ $0.026)$ patients; and categorial fluency scores with all VEP latency components in PDD patients (N1: $r=-0.394, p=0.023, \mathrm{P} 1: r$ $=-0.396, p=0.023, \mathrm{~N} 2: r=-0.565, p=0.001)$ followed with latency significance in PDD-CVH patients (P1: $r=-0.541, p=$ $0.030, \mathrm{~N} 2: r=-0.652, p=0.006)$ and PDD-NCVH patients $(\mathrm{N} 2$ : $r=-0.619, p=0.008)$.

In PDD-CVH patients, significant negative correlation was found for N2 amplitude with the duration of PD $(r=-0.536$, 
TABLE 2 | Comparison of the visual evoked potential components across groups [grouping with any visual hallucination (VH)].

\begin{tabular}{|c|c|c|c|c|c|}
\hline Component & & Controls $(n=21)$ & PDD-NVH $(n=11)$ & PDD-VH $(n=23)$ & Statistics $F, p$-value, $\eta^{2}$ \\
\hline \multirow[t]{2}{*}{ N1 } & Amplitude $(\mu v)$ & $-1.27 \pm 0.93$ & $-1.14 \pm 0.85$ & $-0.70 \pm 0.53$ & $2.78,0.072,0.100$ \\
\hline & Latency (ms) & $88.28 \pm 8.62$ & $90.61 \pm 10.41$ & $92.35 \pm 9.38$ & $0.998,0.376,0.038$ \\
\hline \multirow[t]{2}{*}{ P1 } & Amplitude $(\mu \mathrm{v})$ & $3.61 \pm 2.55$ & $2.82 \pm 1.93$ & $2.11 \pm 1.35$ & $2.425,0.099,0.088$ \\
\hline & Latency (ms) & $124.50 \pm 7.32$ & $128.76 \pm 6.56$ & $126.47 \pm 7.17$ & $1.978,0.149,0.073$ \\
\hline \multirow[t]{2}{*}{ N2 } & Amplitude $(\mu v)$ & $-1.64 \pm 1.44$ & $-1.17 \pm 0.88$ & $-1.09 \pm 1.10$ & $0.843,0.436,0.033$ \\
\hline & Latency (ms) & $162.27 \pm 8.97$ & $174.33 \pm 10.84$ & $176.12 \pm 16.61$ & $7.787, \mathbf{0 . 0 0 1}^{*}, 0.238$ \\
\hline
\end{tabular}

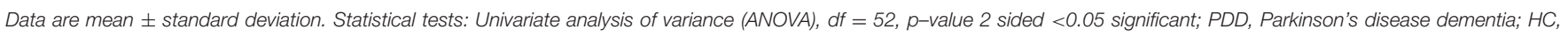

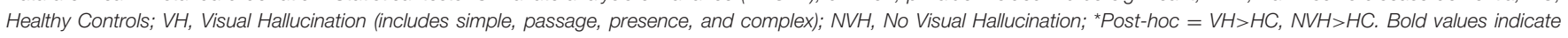
statistically significant values.

TABLE 3 | Correlation of visual evoked potential components (N1, P1, and N2 Latency) with clinical variables.

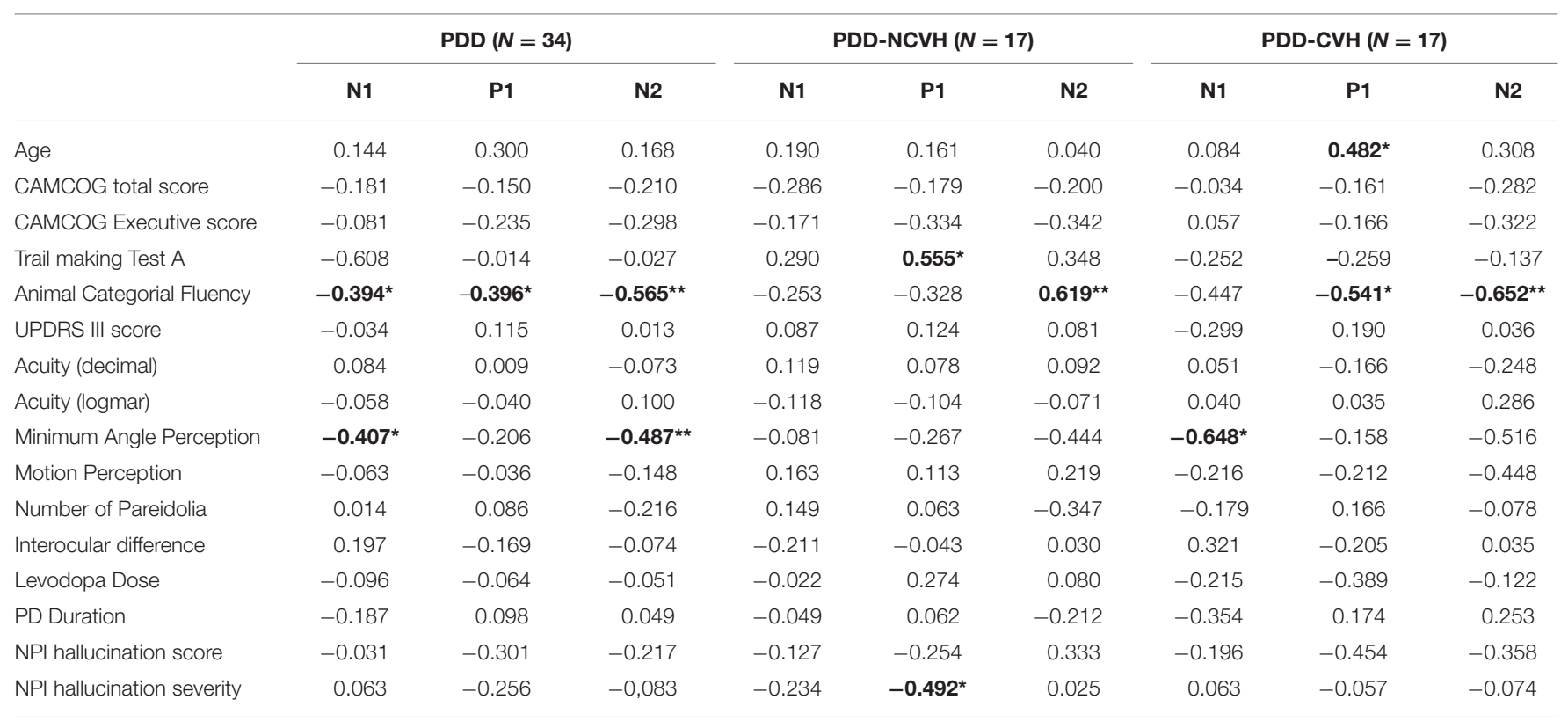

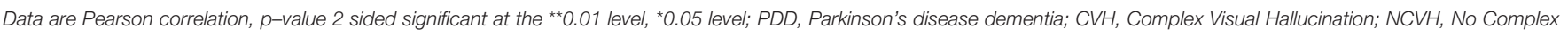
Visual Hallucination. Bold values indicate statistically significant values.

$p=0.026)$ and $\mathrm{P} 1$ latency with age $(r=0.482, p=0.050)$. Correlation analysis with regrouping into $\mathrm{PDD}-\mathrm{NVH}$ and PDD-VH as shown in Table 5 do not change the direction of our results.

\section{DISCUSSION}

In healthy participants, the VEP reflects a combination of many pre-striate and cortical processes. It is noted that a decline in visual pathway integrity following structural changes to the retina can affect the latency and amplitude $(16,49-51)$. In earlier studies the VEP has consistently been shown to be affected by PD neuropathology, indicating substantial decline in the quality of bottom-up visual processing $(3,14,16)$. Following the hypothesis that disrupted bottom-up processing of visual input is associated with the generation of $\mathrm{VH}$ in PDD we investigated whether the VEP could be used as a marker of hallucination symptomology.

In accordance with previous research $(3,27,52-54)$ the PDD patients demonstrated a reduction in visual acuity, impaired visual perception, impoverished motor ability, and compromised global cognition. Analysis of the pattern reversal VEP data revealed a significant increase in the PDD N2 latency relative to controls, especially in PDD-CVH, and non-significant reduction in the PDD P1 amplitude.

P1 and N2 (N140) are both linked to physical properties of the stimulus such as luminance, brightness, position on the retina, and associated attentional demands (55-60). Further, the N2 (N140) has been reported to be associated with increased disease severity (61). This is reflected in our significant correlation of N2 amplitude with the duration of PD in PDD with complex VH.

Significant differences in the interocular latency between the $\mathrm{CVH}$ and $\mathrm{NCVH}$ suggest a difference in low level visual processing between the two groups. Intact low-level visual processing is required to differentiate the different types of inputs projected onto the retina. These interocular differences might be explained by changes within the eye, such as retinal thinning, which is well documented in PD patients (and more prominent in those that experience $\mathrm{VH}$ ) (62). It is known that processing 
TABLE 4 | Correlation of visual evoked potential components (N1, P1 and N2 Amplitude) with clinical variables.

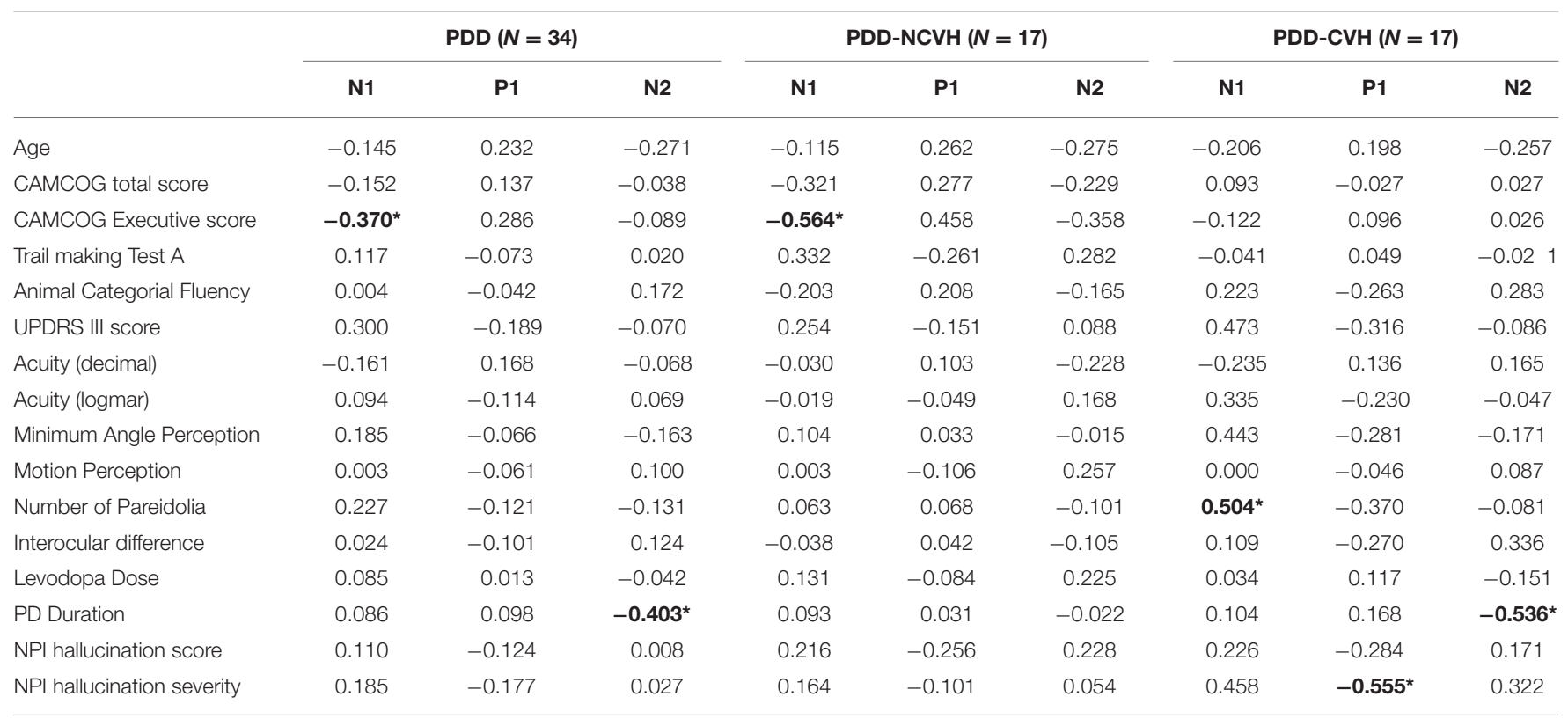

Data are Pearson correlation, $p$-value 2 sided significant at the ${ }^{* *} 0.01$ level, ${ }^{*} 0.05$ level; PDD, Parkinson's disease dementia; CVH, Complex Visual Hallucination; NCVH, No Complex Visual Hallucination. Bold values indicate statistically significant values.

delays between the two eyes can result in an illusory percept (63). Our results thus support greater asymmetry of ocular processing to be involved in CVH. However, no significant correlations were found with the NPI hallucination severity. In future, this is a feature worth studying in more detail, including computational and animal models of PDD macular degeneration and $\mathrm{VH}$.

In patients with PDD there are often abnormalities associated with the structure and function of the retina, including changes in morphology and dopaminergic signaling (3), which have previously been linked to reduced conduction velocity in early visual processing $(16,64-69)$. Source localization of these components places the generating sources deep within the secondary visual cortex $(70,71)$; although their cognitive associations suggest that their activity is governed as part of a higher order visual processing network.

However, our findings are at odds with our hypothesis based on previous work of increased P1 latency (23) in PD with $\mathrm{VH}$. This discrepancy may have arisen because the groups in Matsui et al. were matched on cognitive scores, whereas our two PDD groups did differ on cognitive measures, with more impairment in the PDD-CVH group including perceptual impairment. This is supported by NPI hallucination severity correlating with P1 latency in PDD-NCVH patients and $\mathrm{P} 1$ amplitude in PDD-CVH patients $(r=-0.555, p=0.021)$. The inclusion of minor VH into the PDD-NCVH group potentially explains the correlation with P1 amplitude. Moreover, attentional and perceptual measures (angle detection and pareidolia task) were related to N1 latency and $\mathrm{N} 1$ amplitude in patients with complex VH. These reinforce the argument that whilst bottom up dysfunction places individual at risk of $\mathrm{VH}$, it is disruption of these top down processes which are needed for the manifestation of hallucinations.
Given the extent of association between the VEP components and major clinical measurements in our study, it is clear that there is a deliberate pattern of communication that occurs between the primary visual cortex and both its bottom-up and top-down projections. However, our experimental design is limited in the scope to which we can draw conclusions on the nature of pathological change within the executive system and the link between attention and passive perception of the VEP stimulus.

In the context of a mechanism for $\mathrm{VH}$, our sample results suggest that bottom-up processing is not differentially affected between hallucinators and non-hallucinators. This is not unexpected as it follows that in an integrative model of $\mathrm{VH}$ we would expect $\mathrm{VH}$ content to stem from the interaction of impaired bottom-up processing with dysfunctional topdown control of perception. In our data, complex VH were associated with greater decline in CAMCOG and UPDRS scores, as well as increased numbers of pareidolia relative to patients without complex $\mathrm{VH}$. The divergence in the cognitive and perceptual profile of the groups supports a deteriorated capacity for effective top-down control, which in this model would be a pre-requisite factor for the generation of complex VH. However, these measures were not significantly correlated with the amplitude or latency of the VEP component measurements suggesting that conduction velocity and basic processing of visual feature information is unimpeded by the integrity of detailed perceptual processing. Our findings suggest that the integrity of visual processing has a complex interaction with the higher perceptual function. To build a stronger mechanistic model of this interaction, future work should study the VEP at its cortical sources, whilst 
TABLE 5 | Correlation of visual evoked potential components (N1, P1 and N2) with clinical variables (patients with VH and NVH).

\begin{tabular}{|c|c|c|c|c|c|c|c|c|c|c|c|c|}
\hline & \multicolumn{6}{|c|}{ Latency } & \multicolumn{6}{|c|}{ Amplitude } \\
\hline & N1 & P1 & N2 & N1 & P1 & N2 & N1 & P1 & N2 & N1 & P1 & N2 \\
\hline Age & 0.190 & 0.062 & 0.081 & 0.118 & $0.424^{\star}$ & 0.208 & -0.165 & 0.385 & -0.470 & -0.141 & 0.123 & -0.193 \\
\hline CAMCOG total score & -0.529 & -0.394 & 0.154 & -0.039 & -0.167 & -0.276 & -0.042 & -0.085 & 0.240 & -0.070 & 0.149 & -0.093 \\
\hline CAMCOG Executive score & -0.060 & -0.050 & 0.373 & -0.065 & -0.369 & $-0.477^{\star}$ & -0.560 & 0.346 & -0.132 & -0.188 & 0.203 & -0.066 \\
\hline Animal Categorial Fluency & -0.340 & -0.343 & -0.136 & $-0.436^{\star}$ & $-0.501^{*}$ & $-0.643^{\star \star}$ & 0.173 & -0.281 & 0.417 & 0.080 & -0.063 & 0.154 \\
\hline UPDRS III score & 0.149 & 0.398 & 0.118 & -0.172 & 0.112 & -0.043 & 0.194 & -0.073 & -0.054 & 0.232 & -0.154 & -0.105 \\
\hline Acuity (decimal) & 0.307 & 0.058 & 0.248 & -0.040 & 0.005 & -0.179 & -0.101 & 0.306 & -0.489 & -0.273 & 0.116 & 0.083 \\
\hline Acuity (logmar) & -0.262 & -0.089 & -0.202 & 0.079 & -0.046 & 0.225 & 0.044 & -0.212 & 0.393 & 0.225 & -0.093 & -0.062 \\
\hline Minimum Angle Perception & -0.171 & 0.245 & 0.187 & $-0.500^{\star}$ & -0.230 & $-0.597^{\star *}$ & $0.740^{*}$ & $-0.804^{\star \star}$ & $0.779^{\star}$ & 0.087 & 0.083 & -0.282 \\
\hline PD Duration & 0.096 & 0.031 & -0.134 & -0.330 & 0.182 & 0.066 & -0.002 & 0.081 & -0.065 & 0.002 & 0.218 & $-0.516^{\star}$ \\
\hline NPI hallucination score & -0.450 & -0.042 & 0.054 & -0.061 & -0.279 & -0.314 & -0.106 & 0.107 & -0.085 & -0.087 & -0.040 & -0.006 \\
\hline NPI hallucination severity & -0.450 & -0.042 & 0.054 & 0.144 & -0.259 & -0.229 & -0.106 & 0.107 & -0.085 & -0.052 & -0.093 & 0.015 \\
\hline
\end{tabular}

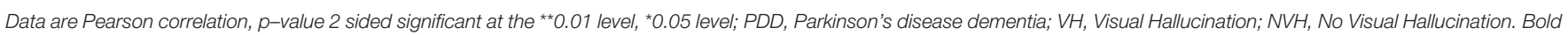
values indicate statistically significant values.

also incorpating structural and functional magnetic resonance imaging data.

Within the integrative model of complex $\mathrm{VH}$ in Lewy body dementias the importance of bottom-up processing is thought to be its influence on the generation of proto-objects $(17,18)$. The frequency and phenomenology of the $\mathrm{VH}$ would then depend on the interaction between the executive system and the perceptual processing centers. Therefore, declining visual health and perceptual quality might simply place the individual in an at-risk state for $\mathrm{VH}$ development (40) rather than directly impact their generation. However, from the results presented we can gleam that Lewy body disorders play a substantial role in the generation of visual hallucinations. This is demonstrated by relationships with both cognition when vision is controlled for, and vice-versa. The lack of a substantive direction for the relationship between $\mathrm{VH}$ complexity and bottom-up and topdown factors suggests that the risk for $\mathrm{VH}$ development is, therefore, more complicated than we had hypothesized. Future research will be required to provide a detailed assessment of the relative contributions of these factors and will require a study design that deliberately varies the cognitive and visual profiles across samples.

\section{Limitations}

There are several limitations. Firstly, the sample size within this study was relatively small, which does not allow for strong conclusions. In future, we will use the data to design larger confirmatory studies. Secondly, we used only a single subjective measure for VH severity. The NPI items are typically collected from the carers of the patient, and do not ask questions about the content of the hallucination. It thus remains possible that there could be a relationship between visual health, bottomup processing, and $\mathrm{VH}$ content that could be accessed by quantifying a scale such as the North East Visual Hallucination Interview (NEVHI) (38). Furthermore, the range of VH severity scores in our groups was limited making correlative analyses more difficult.

\section{CONCLUSION}

In summary, PDD patients demonstrated a diminished profile for visual information processing by way of lowered acuity and reduced visual integrity. This was partially reflected in the outcome of the VEP components, although the broad lack of significant differences between PDD-CVH, PDD-NCVH, and healthy controls implies that bottom-up visual information processing remains reasonably intact. Our findings suggest that while bottom-up processing is not grossly affected by the stage of PDD there is a complex interaction between cognitive, visual, and physiological aspects of visual processing in the generation of VH. To advance our understanding in this field, our findings also support a separation between bottom-up information processing and the mechanism of complex $\mathrm{VH}$ generation, and instead imply that the reduced visual integrity might act to place the individual in an at risk state for the development of hallucinations in patients with a deteriorated cognitive profile. Thus, a main contribution to this field of work, lies in redirecting research from low level visual dysfunction to higher level processes. Future work should focus on a multimodal approach to understanding the interactions between top-down 
and bottom-up perceptual circuitry and how this is impacted by PDD neuropathology.

\section{DATA AVAILABILITY STATEMENT}

The raw data supporting the conclusions of this article will be made available by the authors, without undue reservation.

\section{ETHICS STATEMENT}

The studies involving human participants were reviewed and approved by Newcastle National Health Service (NHS) Health Research Authority (HRA). The patients/participants provided their written informed consent to participate in this study.

\section{AUTHOR CONTRIBUTIONS}

NM, SG, LR, MRB, CA, DC, and J-PT contributed to the conception and organization of the research. NM and AK participated in the execution and data collection. NM, PU, and MF designed and implemented the data analysis

\section{REFERENCES}

1. Biousse V, Skibell BC, Watts RL, Loupe DN, Drews-Botsch C, Newman NJ. Ophthalmologic features of Parkinson's disease. Neurology. (2004) 62:177-80. doi: 10.1212/01.WNL.0000103444.45882.D8

2. Davidsdottir S, Cronin-Golomb A, Lee A. Visual and spatial symptoms in Parkinson's disease. Vision Res. (2005) 45:1285-96. doi: 10.1016/j.visres.2004.11.006

3. Archibald NK, Clarke MP, Mosimann UP, Burn DJ. The retina in Parkinson's disease. Brain. (2009) 132(Pt. 5):1128-45. doi: 10.1093/brain/awp068

4. Urwyler P, Nef T, Killen A, Collerton D, Thomas A, Burn D, et al. Visual complaints and visual hallucinations in Parkinson's disease. Parkinsonism Relat Disord. (2014) 20:318-22. doi: 10.1016/j.parkreldis.2013.12.009

5. Weil RS, Schrag AE, Warren JD, Crutch SJ, Lees AJ, Morris HR. Visual dysfunction in Parkinson's disease. Brain. (2016) 139:2827-43. doi: 10.1093/brain/aww175

6. Aarsland D, Larsen JP, Karlsen K, Lim NG, Tandberg E. Mental symptoms in Parkinson's disease are important contributors to caregiver distress. Int J Geriatr Psychiatry. (1999) 14:866-74.

7. Fénelon G, Mahieux F, Huon R, Ziegler M. Hallucinations in Parkinson's disease: prevalence, phenomenology and risk factors. Brain. (2000) 123:73345. doi: 10.1093/brain/123.4.733

8. McKeith IG, Dickson DW, Lowe J, Emre M, O’Brien JT, Feldman $\mathrm{H}$, et al. Diagnosis and management of dementia with Lewy bodies: third report of the DLB Consortium. Neurology. (2005) 65:1863-72. doi: 10.1212/01.wnl.0000187889.17253.b1

9. Aarsland D, Andersen K, Larsen JP, Lolk A, Kragh-Sorensen P. Prevalence and characteristics of dementia in Parkinson disease: an 8-year prospective study. Arch Neurol. (2003) 60:387-92. doi: 10.1001/archneur.60.3.387

10. Goetz CG, Stebbins GT. Risk factors for nursing home placement in advanced Parkinson's disease. Neurology. (1993) 43:2227-9. doi: 10.1212/WNL.43.11.2227

11. Goetz CG, Stebbins GT. Mortality and hallucinations in nursing home patients with advanced Parkinson's disease. Neurology. (1995) 45:669-71. doi: 10.1212/WNL.45.4.669

12. Nguyen-Legros J. Functional neuroarchitecture of the retina: hypothesis on the dysfunction of retinal dopaminergic circuitry in Parkinson's and interpretation. NM and PU wrote the first draft of the manuscript. RG implemented the data analysis and interpretation for the revision. All authors approved the final version of this manuscript.

\section{FUNDING}

The research was supported by a Swiss National Science Foundation grant (IZK0Z3_173146) to PU and by National Institute for Health Research (NIHR) Newcastle Biomedical Research Centre (BRC) based at Newcastle upon Tyne Hospitals NHS Foundation Trust and Newcastle University. SG was funded by the NIHR MedTech In Vitro Diagnostics Co-operative scheme (ref MIC-2016-014).

\section{ACKNOWLEDGMENTS}

We would like to thank all participants for taking part. The authors are grateful to the group of Professor Etsuro Mori, Tohoku University School of Medicine, Sendai, Japan, for providing a copy of the pareidolia task. This article is currently available as a pre-print on Medrxiv (https://www.medrxiv.org/ content/10.1101/2020.06.28.20142042v1). disease. Surg Radiol Anat. (1988) 10:137-44. doi: 10.1007/BF02 307822

13. Lee JY, Kim JM, Ahn J, Kim HJ, Jeon BS, Kim TW. Retinal nerve fiber layer thickness and visual hallucinations in Parkinson's Disease. Mov Disord. (2014) 29:61-7. doi: 10.1002/mds.25543

14. Bodis-Wollner I, Yahr MD. Measurements of visual evoked potentials in Parkinson's disease. Brain. (1978) 101:661-71. doi: 10.1093/brain/101.4.661

15. Calzetti S, Franchi A, Taratufolo G, Groppi E. Simultaneous VEP and PERG investigations in early Parkinson's disease. J Neurol Neurosurg Psychiatry. (1990) 53:114-7. doi: 10.1136/jnnp.53.2.114

16. Nowacka B, Lubinski W, Honczarenko K, Potemkowski A, Safranow K. Bioelectrical function and structural assessment of the retina in patients with early stages of Parkinson's disease (PD). Doc Ophthalmol. (2015) 131:95-104. doi: 10.1007/s10633-015-9503-0

17. Collerton D, Perry E, McKeith I. Why people see things that are not there: a novel Perception and Attention Deficit model for recurrent complex visual hallucinations. Behav Brain Sci. (2005) 28:737-57; discussion 757-94. doi: 10.1017/S0140525X05000130

18. Shine JM, Halliday GM, Naismith SL, Lewis SJ. Visual misperceptions and hallucinations in Parkinson's disease: dysfunction of attentional control networks? Mov Disord. (2011) 26:2154-9. doi: 10.1002/mds. 23896

19. Onofrj M, Thomas A, Bonanni L. New approaches to understanding hallucinations in Parkinson's disease: phenomenology and possible origins. Expert Rev Neurother. (2007) 7:1731-50. doi: 10.1586/14737175.7.12.1731

20. Fenelon G. Psychosis in Parkinson's disease: phenomenology, frequency, risk factors, and current understanding of pathophysiologic mechanisms. CNS Spectr. (2008) 13(Suppl. 4):18-25. doi: 10.1017/S10928529000 17284

21. Llebaria G, Pagonabarraga J, Martinez-Corral M, Garcia-Sanchez C, PascualSedano B, Gironell A, et al. Neuropsychological correlates of mild to severe hallucinations in Parkinson's disease. Mov Disord. (2010) 25:2785-91. doi: $10.1002 / \mathrm{mds} .23411$

22. Sanchez-Castaneda C, Rene R, Ramirez-Ruiz B, Campdelacreu J, Gascon J, Falcon C, et al. Frontal and associative visual areas related to visual hallucinations in dementia with Lewy bodies and Parkinson's disease with dementia. Mov Disord. (2010) 25:615-22. doi: 10.1002/mds.22873 
23. Matsui H, Udaka F, Tamura A, Oda M, Kubori T, Nishinaka K, et al. The relation between visual hallucinations and visual evoked potential in Parkinson disease. Clin Neuropharmacol. (2005) 28:79-82. doi: 10.1097/01.wnf.0000157066.50948.65

24. Haider M, Spong P, Lindsley DB. Attention, vigilance, and cortical evoked-potentials in humans. Science. (1964) 145:180-2. doi: $10.1126 /$ science.145.3628.180

25. Luck, , S. J. (2005). An Introduction to the Event-Related Potential Technique. Cambridge, MA: The MIT Press, Massachusetts Institute of Technology.

26. Taylor JP, Firbank M, Barnett N, Pearce S, Livingstone A, Mosimann U, et al. Visual hallucinations in dementia with Lewy bodies: transcranial magnetic stimulation study. Br J Psychiatry. (2011) 199:492-500. doi: 10.1192/bjp.bp.110.090373

27. Emre M, Aarsland D, Brown R, Burn DJ, Duyckaerts C, Mizuno $\mathrm{Y}$, et al. Clinical diagnostic criteria for dementia associated with Parkinson's disease. Mov Disord. (2007) 22:1689-707. doi: 10.1002/mds. 21507

28. Folstein MF, Folstein SE, McHugh PR. "Mini-mental state". A practical method for grading the cognitive state of patients for the clinician. J Psychiatr Res. (1975) 12:189-98. doi: 10.1016/0022-3956(75)90026-6

29. Roth M, Tym E, Mountjoy CQ, Huppert FA, Hendrie H, Verma S, et al. CAMDEX. A standardised instrument for the diagnosis of mental disorder in the elderly with special reference to the early detection of dementia. $\mathrm{Br} J$ Psychiatry. (1986) 149:698-709. doi: 10.1192/bjp.149.6.698

30. Roth M, Huppert FA, Tym E, Mountjoy CQ. CAMDEX: The Cambridge Examination for Mental Disorders of the Elderly. Cambridge: Cambridge University Press (1988)

31. Fahn S, Elton R, Committee MOTUD. The Unified Parkinson's disease rating scale. In: Fahn S, Marsden CD, Calne DB, Goldstein M, editors. Recent Developments in Parkinson's Disease. Florham Park, NJ: Macmillan Health Care Information (1987). p. 153-63.

32. Lezak MD, Howieson DB, Loring DW, Hannay HJ, Fischer JS. Neuropsychological Assessment. 4th ed. New York, NY: Oxford University Press (2004).

33. Reitan RM. Validity of the Trail Making test as an indicator of organic brain damage. Percept Mot Skills. (1958) 8:271-6. doi: 10.2466/pms.1958.8.3.271

34. Bach M. The Freiburg Visual Acuity test-automatic measurement of visual acuity. Optom Vis Sci. (1996) 73:49-53. doi: 10.1097/00006324-199601000-00008

35. Wood JS, Firbank MJ, Mosimann UP, Watson R, Barber R, Blamire $\mathrm{AM}$, et al. Testing visual perception in dementia with Lewy bodies and Alzheimer disease. Am J Geriatr Psychiatry. (2013) 21:501-8. doi: 10.1016/j.jagp.2012.11.015

36. Uchiyama M, Nishio Y, Yokoi K, Hirayama K, Imamura T, Shimomura T, et al. Pareidolias: complex visual illusions in dementia with Lewy bodies. Brain. (2012) 135:2458-69. doi: 10.1093/brain/aws126

37. Cummings JL, Mega M, Gray K, Rosenberg-Thompson S, Carusi DA, Gornbein J. The Neuropsychiatric Inventory: comprehensive assessment of psychopathology in dementia. Neurology. (1994) 44:2308-14. doi: 10.1212/WNL.44.12.2308

38. Mosimann UP, Collerton D, Dudley R, Meyer TD, Graham G, Dean JL, et al. A semi-structured interview to assess visual hallucinations in older people. Int J Geriatr Psychiatry. (2008) 23:712-8. doi: 10.1002/gps.1965

39. Urwyler P, Nef T, Muri RM, Killen A, Collerton D, Burn D, et al. Patient and informant views on visual hallucinations in Parkinson disease. Am J Geriatr Psychiatry. (2015) 23:970-6. doi: 10.1016/j.jagp.2014.12.190

40. Firbank MJ, Parikh J, Murphy N, Killen A, Allan CL, Collerton D, et al. Reduced occipital GABA in Parkinson disease with visual hallucinations. Neurology. (2018) 91:e675-85. doi: 10.1212/WNL.0000000000006007

41. Odom JV, Bach M, Brigell M, Holder GE, McCulloch DL, Tormene AP, et al. ISCEV standard for clinical visual evoked potentials (2009 update). Doc Ophthalmol. (2010) 120:111-9. doi: 10.1007/s10633-009-9195-4

42. Oostenveld R, Praamstra P. The five percent electrode system for highresolution EEG and ERP measurements. Clin Neurophysiol. (2001) 112:713-9. doi: 10.1016/S1388-2457(00)00527-7

43. Delorme A, Makeig S. EEGLAB: an open source toolbox for analysis of singletrial EEG dynamics including independent component analysis. J Neurosci Methods. (2004) 134:9-21. doi: 10.1016/j.jneumeth.2003.10.009
44. Lopez-Calderon J, Luck SJ. ERPLAB: an open-source toolbox for the analysis of event-related potentials. Front Hum Neurosci. (2014) 8:213. doi: 10.3389/fnhum.2014.00213

45. Kayser J, Tenke CE. Principal components analysis of Laplacian waveforms as a generic method for identifying ERP generator patterns: I. Evaluation with auditory oddball tasks. Clin Neurophysiol. (2006) 117:348-68. doi: 10.1016/j.clinph.2005.08.034

46. Perrin F, Pernier J, Bertrand O, Echallier JF. Spherical splines for scalp potential and current density mapping. Electroencephalogr Clin Neurophysiol. (1989) 72:184-7. doi: 10.1016/0013-4694(89) 90180-6

47. Ferree TC. Spline Interpolation of the Scalp EEG. Portland: Electical Geodesics, Inc. (2000).

48. Ferree TC. Spherical splines and average referencing in scalp electroencephalography. Brain Topogr. (2006) 19:43-52. doi: 10.1007/s10548-006-0011-0

49. Bodis-Wollner I, Onofrj M. System diseases and visual evoked potential diagnosis in neurology: changes due to synaptic malfunction. Ann N Y Acad Sci. (1982) 388:327-48. doi: 10.1111/j.1749-6632.1982.tb50800.x

50. Bhaskar PA, Vanchilingam S, Bhaskar EA, Devaprabhu A, Ganesan RA. Effect of L-dopa on visual evoked potential in patients with Parkinson's disease. Neurology. (1986) 36:1119-21. doi: 10.1212/WNL.36.8.1119

51. Miri S, Glazman S, Mylin L, Bodis-Wollner I. A combination of retinal morphology and visual electrophysiology testing increases diagnostic yield in Parkinson's disease. Parkinsonism Relat Disord. (2016) 22(Suppl. 1):S134-7. doi: 10.1016/j.parkreldis.2015.09.015

52. Mosimann UP, Mather G, Wesnes KA, O’Brien JT, Burn DJ, McKeith IG. Visual perception in Parkinson disease dementia and dementia with Lewy bodies. Neurology. (2004) 63:2091-6. doi: 10.1212/01.WNL.0000145764.70698.4E

53. Possin KL. Visual spatial cognition in neurodegenerative disease. Neurocase. (2010) 16:466-87. doi: 10.1080/13554791003730600

54. Landy KM, Salmon DP, Galasko D, Filoteo JV, Festa EK, Heindel WC, et al. Motion discrimination in dementia with Lewy bodies and Alzheimer disease. Neurology. (2015) 85:1376-82. doi: 10.1212/WNL.0000000000002028

55. Van Voorhis ST, Hillyard SA. Visual evoked potentials and selective attention to points in space. Perception and Psychophysics. (1977) 22:54-62. doi: 10.3758/BF03206080

56. Hillyard SA, Munte TF. Selective attention to color and location: an analysis with event-related brain potentials. Percept Psychophys. (1984) 36:185-98. doi: 10.3758/BF03202679

57. Luck SJ, Hillyard SA, Mouloua M, Woldorff MG, Clark VP, Hawkins HL. Effects of spatial cuing on luminance detectability: psychophysical and electrophysiological evidence for early selection. J Exp Psychol Hum Percept Perform. (1994) 20:887-904. doi: 10.1037/0096-1523.20.4.887

58. Johannes S, Munte TF, Heinze HJ, Mangun GR. Luminance and spatial attention effects on early visual processing. Brain Res Cogn Brain Res. (1995) 2:189-205. doi: 10.1016/0926-6410(95)90008-X

59. Ito $M$, Gilbert CD. Attention modulates contextual influences in the primary visual cortex of alert monkeys. Neuron. (1999) 22:593-604. doi: 10.1016/S0896-6273(00)80713-8

60. Johannes S, Wieringa BM, Nager W, Rada D, Muller-Vahl KR, Emrich HM, et al. Tourette syndrome and obsessive-compulsive disorder: event-related brain potentials show similar mechanisms [correction of mechansims] of frontal inhibition but dissimilar target evaluation processes. Behav Neurol. (2003) 14:9-17. doi: 10.1155/2003/326468

61. Talebi M, Meshkinghalam A, Andalib S. Association between visual evoked potential and disease severity, disease duration and visual hallucination in patients with idiopathic parkinsonism. Novel Biomed. (2014) 2:120-5. doi: $10.22037 /$ nbm.v2i4.6696

62. Marques A, Beze S, Pereira B, Chassain C, Monneyron N, Delaby L, et al. Visual hallucinations and illusions in Parkinson's disease: the role of ocular pathology. J Neurol. (2020) 267:2829-41. doi: 10.1007/s00415-020-09925-x

63. Reynaud A, Hess RF. Interocular contrast difference drives illusory 3D percept. Sci Rep. (2017) 7:5587. doi: 10.1038/s41598-017-06151-w

64. Regan D, Neima D. Low-contrast letter charts in early diabetic retinopathy, ocular hypertension, glaucoma, and Parkinson's disease. $\mathrm{Br} J$ Ophthalmol. (1984) 68:885-9. doi: 10.1136/bjo.68.12.885 
65. Bodis-Wollner I, Marx MS, Mitra S, Bobak P, Mylin L, Yahr M. Visual dysfunction in Parkinson's disease. Loss in spatiotemporal contrast sensitivity. Brain. (1987) 110:1675-98. doi: 10.1093/brain/110.6.1675

66. Jones RD, Donaldson IM, Timmings PL. Impairment of high-contrast visual acuity in Parkinson's disease. Mov Disord. (1992) 7:232-8. doi: $10.1002 / \mathrm{mds} .870070308$

67. Price MJ, Feldman RG, Adelberg D, Kayne H. Abnormalities in color vision and contrast sensitivity in Parkinson's disease. Neurology. (1992) 42:887-90. doi: 10.1212/WNL.42.4.887

68. Pieri V, Diederich NJ, Raman R, Goetz CG. Decreased color discrimination and contrast sensitivity in Parkinson's disease. J Neurol Sci. (2000) 172:7-11. doi: 10.1016/S0022-510X(99)00204-X

69. Holroyd S, Currie L, Wooten GF. Prospective study of hallucinations and delusions in Parkinson's disease. J Neurol Neurosurg Psychiatry. (2001) 70:734-8. doi: 10.1136/jnnp.70.6.734

70. Di Russo F, Martinez A, Sereno MI, Pitzalis S, Hillyard SA. Cortical sources of the early components of the visual evoked potential. Hum Brain Mapp. (2002) 15:95-111. doi: 10.1002/hbm.10010
71. Di Russo F, Pitzalis S, Spitoni G, Aprile T, Patria F, Spinelli D, et al. Identification of the neural sources of the pattern-reversal VEP. Neuroimage. (2005) 24:874-86. doi: 10.1016/j.neuroimage.2004.09.029

Conflict of Interest: The authors declare that the research was conducted in the absence of any commercial or financial relationships that could be construed as a potential conflict of interest.

The reviewer DF declared past co-authorship and research collaboration with several of the authors DC, J-PT, PU, MF to the handling Editor.

Copyright (C) 2021 Murphy, Killen, Gupta, Graziadio, Rochester, Firbank, Baker, Allan, Collerton, Taylor and Urwyler. This is an open-access article distributed under the terms of the Creative Commons Attribution License (CC BY). The use, distribution or reproduction in other forums is permitted, provided the original author(s) and the copyright owner(s) are credited and that the original publication in this journal is cited, in accordance with accepted academic practice. No use, distribution or reproduction is permitted which does not comply with these terms. 